\title{
Efeito do treinamento resistido na sobrevida de camundongos c57bl/6 com caquexia associada ao melanoma cutâneo
}

\section{Effect of resisted training on survival of $c 57 \mathrm{bl} / 6$ mice with cachexia associated with cutaneous melanoma}

\author{
Lawrey Vanessa Rocha Soares ${ }^{1}$ \\ João Vitor Nunes Lopes ${ }^{2}$ (1) \\ Elenice Rocha Soares ${ }^{3}$ (1)
}

\author{
Mariana Rocha Alves 4 (1) \\ Vinicius Dias Rodrigues 5
}

${ }^{1}$ Universidade Estadual de Montes Claros (Montes Claros). Minas Gerais, Brasil. lawreyarmario@gmail.com ${ }^{2}$ Autor para correspondência. Universidade Estadual de Montes Claros (Montes Claros). Minas Gerais, Brasil. joaollopes@outlook.com.br 3,5 Universidade Estadual de Montes Claros (Montes Claros). Minas Gerais, Brasil. eleniceroch705@gmail.com, viniciuslabex@hotmail.com ${ }^{4}$ Universidade Federal Fluminense (Niterói). Rio de Janeiro, Brasil. marianarochaalves13@gmail.com

RESUMO | OBJETIVO: O objetivo desse estudo foi verificar o efeito do treinamento resistido na sobrevida de camundongos C57BL/6 com caquexia associada ao melanoma cutâneo. MATERIAL E MÉTODOS: A amostra foi constituída por 64 (camundongos C57BL/6 fêmeas, com idade entre 10 e 12 semanas, com cerca de $50 \pm 5$ gramas de peso corporal. Os camundongos foram dis tribuídos aleatoriamente em quatros grupos: i. camundongos controle, com indução de tumor, inativos fisicamente (Controle, $n=16$ ); ii. camundongos submetidos ao treinamento resistido diário somente antes da indução tumoral (Treino 1, $\mathrm{n}=16$ ); iii. camundongos submetidos ao treinamento resistido diário antes e após da indução tumoral (Treino 2, $\mathrm{n}=16$ ); iv. camundongos submetidos ao treinamento resistido diário após a indução tumoral e apresentado quadro caquético (Treino $3, \mathrm{n}=16$ ). No procedimento para treinamento resistido (TR) com choque, foi utilizada uma escada com $110 \mathrm{~cm}$ de altura, $18 \mathrm{~cm}$ de largura, $2 \mathrm{~cm}$ entre os degraus e 80 graus de inclinação. No procedimento para TR com choque, foi utilizada uma escada $110 \mathrm{~cm}$ de altura, $18 \mathrm{~cm}$ de largura, $2 \mathrm{~cm}$ entre os degraus e 80 graus de inclinação. O exercício resistido baseia-se na subida dos camundongos. Na plataforma de saída, aplicava-se um choque elétrico como estímulo para subir as escadas, nesta etapa era aplicada o choque nas quatro patas do animal com uma tensão elétrica de 20 volts a uma frequência de 45 Hertz durante seis séries de oito repetições, cada uma com noventa segundos de intervalo entre as séries. Os grupos de camundongos foram submetidos a acompanhamento por no máximo 15 dias após o diagnóstico da caquexia a fim de comparar a sobrevida geral relacionada ao câncer entre os grupos de estudo. As curvas de sobrevivência de KaplanMeier foram estimadas para cada evento e as curvas dos diferentes grupos foram comparadas usando o teste de Log-rank. O tempo de sobrevida proposta foi de 25 dias após inoculação. RESULTADOS: Os resultados apresentados nesse estudo mostraram que não houve diferença significativa $(p<0,05)$ entre as propostas de treino. CONCLUSÃO: Não houve diferença na sobrevida de animais com caquexia associada ao modelo tumoral singênico de melanoma cutâneo com intervenção de exercício resistido ou sedentários.

PALAVRAS-CHAVE: Treinamento Resistido. Câncer. Caquexia Relacionada ao Câncer. Modelo Melanoma Cutâneo Singênico. Sobrevida.
ABSTRACT I OBJECTIVE: The objective of this study was to verify the effect of resistance training on the survival of C57BL / 6 mice with cachexia associated with cutaneous melanoma. MATERIAL AND METHODS: The sample consisted of 64 (female C57BL/ 6 mice, aged between 10 and 12 weeks, with approximately $50 \pm 5$ grams of body weight. The mice were randomly distributed into four groups: i. control mice, with tumor induction, physically inactive (Control, $n=16$ ); ii. mice submitted to daily resistance training only before tumor induction (Training $1, n=16$ ); iii. Mice submitted to daily resistance training before and after tumor induction (Training 2, n = 16); iv. Mice submitted to daily resistance training after tumor induction and presented a cachectic condition (Training $3, n=16$ ). In the resistance training procedure (RT) with shock, a $110 \mathrm{~cm}$ high, $18 \mathrm{~cm}$ wide, $2 \mathrm{~cm}$ between the steps, and 80 degrees inclination ladder was used. In the procedure for shock resistance training (TR), a ladder $110 \mathrm{~cm}$ high, $18 \mathrm{~cm}$ wide, $2 \mathrm{~cm}$ between the rungs, and 80 degrees of inclination was used. The resisted exercise is based on the climbing of the mice. On the exit platform, an electric shock was applied as a stimulus to climb the stairs. In this step, the shock was applied to the four legs of the animal with an electric voltage of 20 volts at a frequency of 45 Hertz during six series of eight repetitions, each with ninety-seconds intervals between the series. The mice groups underwent follow-up for no more than 15 days after diagnosis of cachexia to compare overall cancer-related survival between the study groups. Kaplan-Meier survival curves were estimated for each event, and the curves of the different groups were compared using the Log-rank test. The proposed survival time was 25 days after inoculation. RESULTS: The results presented in this study showed no significant difference $(p<0.05)$ between the training proposals. CONCLUSION: There was no difference in animals' survival with cachexia associated with the syngeneic melanoma skin tumor model with either resistance exercise or sedentary intervention.

KEYWORDS: Resistance Training. Cancer. Cancer-Related Cachexia. Cutaneous Singular Melanoma Model. Survival. 


\section{Introdução}

O melanoma é uma neoplasia maligna que se origina nos melanócitos, células da pele produtoras de um pigmento denominado melanina. ${ }^{1}$ Tem início com um pequeno tumor cutâneo pigmentado sobre a pele normal, mais frequentemente em áreas expostas ao sol, mas quase metade dos casos ocorre a partir de nevos melanocitos pré-existentes.?

Melanoma é o mais agressivo câncer de pele, frequentemente mostrando rápido crescimento e disseminação metastática. O melanoma é classificado de quatro maneiras principais, por suas características clínicas e histológicas: melanoma nodular, lentigo maligno melanoma, melanoma sacral e extensivo superficial. ${ }^{3}$ De acordo com Araújo et al. ${ }^{4}$, o melanoma cutâneo maligno constitui cerca de 5\% dos tumores cutâneos malignos, apresentando alta incidência e alta letalidade, sendo responsável pela vasta maioria das mortes por câncer de pele. A sobrevida do melanoma tem aumentado gradativamente nos últimos anos. ${ }^{4}$

A caquexia é associada ao efeito adverso do câncer, reduzindo a função física, a tolerância ao tratamento antineoplásico e a diminuição da sobrevida. ${ }^{5}$ É um tipo de desnutrição especifico e debilitante em pacientes de tumores malignos avançados, em relação a $20 \%$ das mortes em pacientes com câncer. ${ }^{6}$ Segundo Duval et al., , um dos desafios da caquexia no câncer é o controle da perda de peso, relacionada principalmente a alterações metabólicas importantes, à anorexia e redução da ingestão de calorias, lipídeos e proteínas. Considerando os desafios relacionados à perda de peso progressiva com a caquexia associada ao câncer, torna-se importante a identificação precoce de seus fatores desencadeantes para uma intervenção e preservação dessas funções. ${ }^{\underline{7}}$

As adaptações provocadas pelo exercício físico apresentam um impacto na prevenção de diversas doenças; dentre elas, o câncer. $\underline{2}$ A atividade e o exercício físico trazem inúmeros benefícios e para o indivíduo8. De acordo com Santos et al.. , o treinamento em geral tem a capacidade de induzir mudanças positivas tanto na via de síntese, quanto na degradação proteica, já que ambas as vias estão relacionadas à perda de tecido muscular em indivíduos oncológicos.

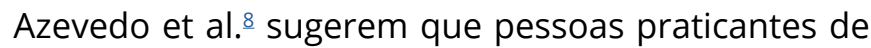
exercício físico têm um maior vigor possível em relação às funções em diversas atividades, aumentando a qualidade de vida à medida que envelhecem; por consequência, aumentando a expectativa de vida. Dentre as diversas possibilidades de treinamento, o TR é apresentado como uma proposta positiva no tratamento da caquexia associada ao câncer, o TR provoca adaptações neuromusculares e hipertróficas que podem contribuir com a melhora dessa situação patológica. $\frac{10}{}$

Somando os benefícios do exercício físico na expectativa de vida com a situação da caquexia associada ao câncer, percebe-se que as possíveis respostas podem acarretar melhora na sobrevida nesse quadro complexo. ${ }^{11}$ Dessa forma, o presente estudo tem como objetivo verificar o efeito do treinamento resistido na sobrevida de camundongos C57BL/6 com caquexia associada ao melanoma cutâneo.

\section{Materiais e métodos}

\section{Caracterização da amostra e cuidados éticos}

Estudo caracterizado como experimental, analítico, prospectivo e de abordagem quantitativa. Este estudo foi aprovado pelo Comitê de Ética em experimentação animal e bem-estar da Universidade Estadual de Montes Claros (Unimontes) e teve parecer favorável para execução. A amostra arbitrariamente foi constituída por 64 (camundongos C57BL/6 fêmeas, com idade entre 10 e 12 semanas, com cerca de $50 \pm$ 5 gramas de peso corporal.

\section{Desenho experimental}

Os camundongos foram distribuídos aleatoriamente em quatros grupos: i. camundongos controle, com indução de tumor, inativos fisicamente (Grupo 1, $\mathrm{n}$ = 16); ii. camundongos submetidos ao treinamento resistido diário somente antes da indução tumoral (Grupo 2, $\mathrm{n}=16$ ); iii. camundongos submetidos ao treinamento resistido diário antes e após da indução tumoral (Grupo 3, $n=16$ ); iv. camundongos submetidos ao treinamento resistido diário somente após quadro caquético diagnosticado (Grupo 4, n = 16). 


\section{Diagnóstico da caquexia}

Foi estabelecido o décimo dia para o diagnóstico de caquexia após a indução tumoral para todos os animais. A escolha arbitraria foi em função da literatura cientifica atual na utilização dessa padronização. ${ }^{9}$

\section{Indução tumoral}

Todos os camundongos C57BI/6 foram imobilizados e submetido à tricotomia da região dorsal, próxima ao pescoço, para a indução do tumor. ${ }^{\circledR}$ Logo após, foi verificada a ausência de sensibilidade dolorosa reflexa. 9 Após assepsia com álcool iodado, os animais foram inoculados com 5×105 células de melanoma murino B16-F10, ressuspensas em $50 \mu \mathrm{L}$, na região subcutânea dorsal, próxima à base do pescoço (flanco). ${ }^{9} \mathrm{~A}$ inoculação dessa quantidade de células viáveis na região subcutânea tem a capacidade de concluir um ciclo mitótico em 24 h e de avultar o tumor no prazo de 3-4 dias. .12

\section{Instrumentos e procedimentos do treinamento resistido}

No procedimento para TR com choque, utilizou-se uma escada $110 \mathrm{~cm}$ de altura, $18 \mathrm{~cm}$ de largura, $2 \mathrm{~cm}$ entre os degraus e 80 graus de inclinação. O exercício resistido baseia-se na subida dos camundongos. $\mathrm{Na}$ plataforma de saída, aplicava-se um choque elétrico como estímulo para subir as escadas; nesta etapa, era aplicado o choque nas quatro patas do animal, com uma tensão elétrica de 20 volts, a uma frequência de 45 Hertz durante seis séries de oito repetições, cada uma com noventa segundos de intervalo entre as séries. $\frac{13}{}$ Na proposta do grupo 2 , ocorreram 15 sessões de ER, na proposta do grupo 3 ocorreram 40 sessões de $E R$, na posposta do grupo 4 ocorreram 15 sessões de ER. A duração da sessão era de 25 minutos.

\section{Análise de sobrevivência}

Os grupos de camundongos foram submetidos a acompanhamento por no máximo 15 dias após o diagnóstico da caquexia, a fim de comparar a sobrevida geral relacionada ao câncer entre os grupos de estudo. No entanto, todos os camundongos foram sacrificados assim que se tornaram moribundos, exibindo grave comprometimento das funções corporais ou comportamento, devido à extensa necrose, ulceração e crescimento da massa tumoral.

A eutanásia ocorreu por luxação cervical. No primeiro momento, os animais foram anestesiados com cetamina $(75 \mathrm{mg} / \mathrm{kg}) / x i l a z i n a(5 \mathrm{mg} / \mathrm{kg})$, respectivamente. $.14,15$ Os tecidos coletados foram pesados usando uma balança digital de precisão analítica (A. Cientifica EEQ9003E). 13,14

\section{Tratamento de dados}

Os dados coletados foram analisados no software SPSS (Statistical Package for the Social Sciences) versão 20.0. O nível de confiança adotado em todas as análises foi fixado em 95\% ( $p<0,05)$. As curvas de sobrevivência de Kaplan-Meier foram estimadas para cada evento e as curvas dos diferentes grupos foram comparadas usando o teste de Log-rank.

\section{Resultados}

Os resultados apresentados nesse estudo mostraram que não houve diferença significativa entre as propostas de treino. O tempo de sobrevida proposta foi de 25 dias após inoculação. 
Figura 1. A análise de sobrevivência dos camundongos C57BL/6 não apresentou diferença significativa entre os tratamentos propostos.

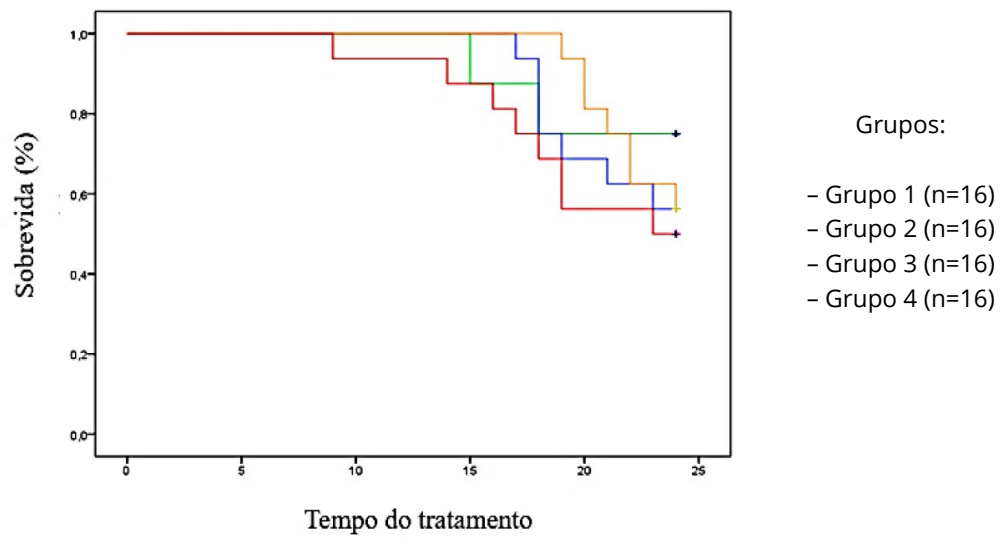

\section{Discussão}

A caquexia no câncer é uma consequência debilitante da progressão da doença, caracterizada pela perda significativa de peso através do catabolismo do músculo esquelético e do tecido adiposo. $\frac{10}{} \mathrm{~A}$ literatura aponta que praticantes de atividade física regularmente têm uma melhor qualidade e expectativa de vida do que aqueles menos ativos. $\frac{16}{}$

O TR, em virtude de seu efeito anti-inflamatório, é demonstrado ser eficaz na neutralização do catabolismo muscular, aumentando a síntese proteica e reduzindo a sua degradação, melhorando com sucesso a força muscular, a função física e a qualidade de vida em pacientes com caquexia não relacionada ao câncer. 10 O TR regula a função imune de sobreviventes de câncer, exercendo um papel anti-inflamatório dos tecidos, reduzindo a concentração das citocinas próinflamatórias TNF-a e IL-6. ${ }^{17}$ Assim, ao implementar intervenções de exercícios adequados, pode ser possível reverter o catabolismo proteico, enquanto aumenta-se a síntese de proteínas e a massa corporal magra, podendo neutralizando a caquexia..$^{10}$ Dessa forma, o exercício físico em geral atua na prevenção do câncer incorporado como parte da prática padrão no cuidado do câncer, visto como uma terapia adjunta que ajuda a neutralizar os efeitos adversos do câncer e seu tratamento. ${ }^{17,18}$

O TR é seguro durante e após os tratamentos de câncer e resulta em melhorias no funcionamento físico, qualidade de vida e fadiga relacionada ao câncer em vários grupos de sobreviventes de câncer. ${ }^{19} \mathrm{~A}$ proposta do treino resistido para roedores é apresentada como alternativa metodológica para estudos, pois apresenta resultados semelhantes com seres humanos $\frac{13}{3}$, mas o modelo proposto nesse estudo de caquexia associada ao câncer foi muito agressivo. Tal situação pode ter sido um fator limitante, pois a sobrevida dos animais foi diferente quando comparado com o grupo 1 (controle), dessa forma, estudos experimentais com outros modelos metodológicos de exercício físico com camundongos C57BL/6 devem ser incentivados para elucidar as lacunas aqui deixadas.

\section{Conclusões}

Nesse contexto, concluímos que não houve diferença na sobrevida de animais com caquexia associada ao modelo tumoral singênico de melanoma cutâneo com intervenção de exercício resistido quando comparados com o grupo controle.

\section{Contribuições dos autores}

Lopes JVN foi responsável pela escrita do artigo, delineamento estatístico. Soares LVR foi responsável pela coleta de dados e manejo animal. Soares ER foi responsável pelo manejo animal. Alves MR foi responsável pelo delineamento estatístico e análise laboratorial. Rodrigues VD foi responsável pela orientação e coordenação geral da pesquisa.

\section{Conflitos de interesses}

Nenhum conflito financeiro, legal ou político envolvendo terceiros (governo, empresas e fundações privadas, etc.) foi declarado para nenhum aspecto do trabalho submetido (incluindo, mas não se limitando a subvenções e financiamentos, participação em conselho consultivo, desenho de estudo, preparação de manuscrito, análise estatística, etc.). 


\section{Referências}

1. Mânica A, Lang MTG. Relação entre o desenvolvimento do melanoma cutâneo e o estresse oxidativo. RBAC [Internet]. 2017;49(1):22-5. Disponível em: http://www.rbac.org.br/wpcontent/uploads/2017/06/RBAC-1-2017-ref.-278.pdf

2. Rodrigues VD. Efeitos do treinamento resistido e da atividade física em camundongos C57BL/6 com caquexia associada ao modelo tumoral singênico de melanoma cutâneo [tese]. Minas Gerais: Universidade Estadual de Montes Claros; 2018.

3. Marques SA, Marques MEA, Espósito ACC. Sinais de alerta nas lesões melanocíticas. Rede Câncer [Internet]. 2013:36-7. Disponível em: https://www.inca.gov.br/sites/ufu.sti.inca.local/ files//media root/rrc-22-artigo-sinais-de-alerta-nas-lesoesmelanociticas.pdf

4. Araujo IC, Coelho CMS, Saliba GAM, Lana PC, Almeida ACM, Pereira NA, et al. Melanoma Cutâneo: aspectos clínicos, epidemiológicos e anatomopatológicos de um centro de formação em Belo Horizonte. Rev. Bras. Cir. Plást. 2014;29(4):497503. http://www.dx.doi.org/10.5935/2177-1235.2014RBCP0088

5. Duval PA, Bergmann RB, Vale IAV, Colling C, Araújo ES, Assunção MCF. Prevalência de caquexia neoplásica e fatores associados na internação domiciliar. Rev. bras. Cancerol [Internet]. 2015;61(3):261-7. Disponível em: https://rbc.inca.gov.br/site/ arquivos/n_61/v03/pdf/09-artigo-prevalencia-de-caquexianeoplasica-e-fatores-associados-na-internacao-domiciliar.pdf

6. Argilés JM, Busquets S, Stemmler B, López-Soriano FJ. Cancer cachexia: understanding the molecular basis. Nat Rev Cancer. 2014;14(11):754-62. https://doi.org/10.1038/nrc3829

7. Lima KS, Lima MCL, Araújo AO, Lima KS, Burgos MGPA, Arruda IKG, et al. Caquexia e pré-caquexia em pacientes com câncer do trato gastrointestinal. Nutr. clín. diet. Hosp. [Internet]. 2017;37(4):101-7. Disponível em: https://dialnet.unirioja.es/ servlet/articulo?codigo $=6636994$

8. Azevedo MAS. O envelhecimento ativo e a qualidade de vida: uma revisão integrativa [dissertação] [Internet]. Portugal: Escola superior de enfermagem do Porto; 2015. Disponível em: https:// comum.rcaap.pt/bitstream/10400.26/10776/1/marta\%2020\%20 de\%20abril\%20-\%20tese\%20final\%20-\%20pdf.pdf

9. Santos K, Oliveira T, Lopes JN, Freitas A, Vieira MM, Sousa BO. Efeito da atividade física em ambiente enriquecido sobre o músculo esquelético de camundongos com caquexia associado ao melanoma cutâneo. Rev Pesqui Fisioter. 2020;10(3):436-41. http://dx.doi.org/10.17267/2238-2704rpf.v10i3.3016

10. Gould DW, Lahart I, Carmichael AR, Koutedakis Y, Metsios GS. Cancer cachexia prevention via physical exercise: molecular mechanisms. J Cachexia Sarcopenia Muscle. 2013;4(2):111-24. https://dx.doi.org/10.1007\%2Fs13539-012-0096-0
11. Loureiro MM, Padrão Al, Duarte JA, Ferreira R. Impacto do exercício físico regular no catabolismo muscular subjacente à caquexia associada ao cancro. Rev. port. ciênc. desporto. 2014;14(2):95-109. http://dx.doi.org/10.5628/rpcd.14.02.95

12. Trunova GV, Makarova OV, Diatroptov ME, Bogdanova IM, Mikchailova LP, Abdulaeva SO. Morphofunctional characteristic of the immune system in BALB/C and C57BL/6 mice. Bull Exp Biol Med. 2011;151(1):99-102. https://doi.org/10.1007/s10517-0111268-1

13. Rodrigues VD, Pimentel DM, Brito AS, Vieira MM, Santos AR, Machado AS, et al. Methodological validation of a vertical ladder with low intensity shock stimulus for resistance training in C57BL/6 mice: Effects on muscle mass and strength, body composition, and lactate plasma levels. J Hum Sport Exerc. 2019;14(3):608-31. https://doi.org/10.14198/jhse.2019.143.12

14. Voltarelli FA, Frajacomo FT, Padilha CS, Testa MTJ, Cella PS, Ribeiro DF, et al. Syngeneic B16F10 Melanoma Causes Cachexia and Impaired Skeletal Muscle Strength and Locomotor Activity in Mice. Front Physiol. 2017;8:715. https://dx.doi. org/10.3389\%2Ffphys.2017.00715

15. Honors MA, Kinzig KP. Characterization of the Yoshida sarcoma: a model of cancer cachexia. Support Care Cancer. 2013;21(10):2687-94. https://doi.org/10.1007/s00520-013-1839-y

16. Tolentino GP, Battaglini CL, Conde DM, Araujo SS, Santana A, Oliveira RJ, et al. Câncer de mama e exercício físico. Rev Bras Med [Internet]. 2010;67(3):78-81. Disponível em: https://www.researchgate.net/profile/Grassyara-Tolentino/ publication/287518517_Breast_cancer_and_physical_exercise/ links/586535ab08ae329d620456b7/Breast-cancer-and-physicalexercise.pdf

17. Lima FD. Atividade física e câncer [Internet]. In: Santos M, Corrêa TS, Faria LDBB, Reis PED, Pinheiro RN. Diretrizes Oncológicas 2. São Paulo: Doctor Press; 2019. Disponível em: https://diretrizesoncologicas.com.br/wp-content/ uploads/2018/10/Diretrizes-oncol\%C3\%B3gicas-2_Parte47.pdf

18. Cormie P, Atkinson M, Bucci L, Cust A, Eakin E, Hayes S, et al. Clinical Oncology Society of Australia position statement on exercise in cancer care. Med J Aust. 2018;209(4):184-7. https://doi. org/10.5694/mja18.00199

19. Schmitz KH, Courneya KS, Matthews C, Demark-Wahnefried W, Galvão DA, Pinto BM, et al. American College of Sports Medicine roundtable on exercise guidelines for cancer survivors. Med Sci Sports Exerc. 2010;42(7):1409-26. https://doi.org/10.1249/ mss.0b013e3181e0c112 